\title{
Cárie na infância: epidemiologia, etiologia e prevenção.
}

\author{
Moizes Bozano de Souza (1) e Francisca Carla Benitez de Paula (1)
}

\section{ARTIGO DE REVISÃO}

Resumo

A cárie na primeira infância (CPI) é uma das doenças mais prevalentes em crianças em todo o mundo. A CPI é impulsionada por um estado disbiótico dos microrganismos orais causado principalmente por uma dieta rica em açúcar. Além disso, a má higiene oral ou a remoção insuficiente da placa dentária levam à rápida progressão da CPI. A CPI não só leva à destruição dentária e dor nas crianças, mas também afeta a qualidade de vida dos cuidadores. Crianças com CPI extensa apresentam alto risco de desenvolver cárie com a dentição permanente ou terão outros problemas para falar e / ou comer. Para prevenir a CPI, várias estratégias devem ser levadas em consideração. As crianças devem escovar os dentes com dentifrícios que contenham ingredientes suaves, como surfactantes suaves e agentes que apresentem propriedades antiaderentes em relação aos microrganismos orais. Os pais / responsáveis devem ajudar seus filhos a escovar os dentes. Além disso, agentes remineralizantes e não tóxicos devem ser incluídos na formulação do creme dental. Dois agentes biomiméticos promissores para higiene bucal de crianças são fosfato de cálcio amorfo $\left[\mathrm{Ca}_{x}\left(\mathrm{PO}_{4}\right)_{y} n \mathrm{H}_{2} \mathrm{O}\right]$ e hidroxiapatita $\left[\mathrm{Ca}_{5}\left(\mathrm{PO}_{4}\right)_{3}(\mathrm{OH})\right]$.

Palavras-chave: Cárie; Primeira infância; Odontopediatria, Epidemiologia. 


\title{
Early Childhood Caries: Epidemiology, Aetiology, and Prevention.
}

\begin{abstract}
Early childhood caries (ECC) is one of the most prevalent diseases in children worldwide. ECC is driven by a dysbiotic state of oral microorganisms mainly caused by a sugar-rich diet. Additionally, poor oral hygiene or insufficient dental plaque removal leads to the rapid progression of ECC. ECC leads not only to dental destruction and pain with children, but also affects the quality of life of the caregivers. Children with extensive ECC are at high risk to develop caries with the permanent dentition or will have other problems with speaking and/or eating. To prevent ECC, several strategies should be taken into account. Children should brush their teeth with toothpastes containing gentle ingredients, such as mild surfactants and agents showing antiadherent properties regarding oral microorganisms. Parents/caregivers have to help their children with brushing the teeth. Furthermore, remineralizing and nontoxic agents should be included into the toothpaste formulation. Two promising biomimetic agents for children's oral care are amorphous calcium phosphate $\left[\mathrm{Ca}_{x}\left(\mathrm{PO}_{4}\right)_{y} n \mathrm{H}_{2} \mathrm{O}\right]$ and hydroxyapatite $\left[\mathrm{Ca}_{5}\left(\mathrm{PO}_{4}\right)_{3}(\mathrm{OH})\right]$.
\end{abstract}

Keywords: Caries; Early childhood; Pediatric Dentistry, Epidemiology.

Instituição afiliada: 1- Grupo de pesquisa em odontologia clínica, Goiania, Goiais, Brasil.

Dados da publicação: Artigo recebido em 10 de junho, revisado em 15 de junho, aceito para publicação em 20 de junho e publicado em 30 de junho.

DOI: https://doi.org/10.36557/2674-8169.2021v3n6p30-48

Moizes Bozano de Souza dr.momoca34@gmail.com

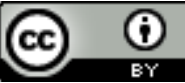

This work is licensed under a Creative Commons Attribution 4.0 International

License. 


\section{INTRODUÇÃO}

A cárie na primeira infância $(\mathrm{CPI})$ ainda é uma das doenças mais prevalentes em crianças em todo o mundo. A CEC não afeta apenas a saúde bucal das crianças, mas também a saúde geral das crianças [ 1 , 2 ]. Não apenas dor oral, problemas ortodônticos e defeitos de esmalte, mas também problemas com a alimentação e a fala podem ocorrer, bem como um risco aumentado de desenvolvimento de cárie na dentição permanente [3]. A perda prematura da dentição decídua frequentemente leva a problemas ortodônticos na vida adulta [ 4 ]. Não apenas as crianças são afetadas, mas também os pais serão influenciados por esta doença, sendo os cuidadores responsáveis [ 3 , 4] Por exemplo, os problemas dentários foram apontados como os principais motivos de hospitalização de crianças na Austrália em 2015 [ 5 ]. Assim, a CPI leva não apenas à dor temporária, mas, mais importante, tem efeitos importantes na qualidade de vida da família / cuidadores, incluindo implicações financeiras e de saúde [6,7]. O objetivo deste artigo de revisão é apresentar o estado da arte da epidemiologia, etiologia, características da dentição decídua, fatores de risco, recomendações gerais e estratégias de prevenção de CPI.

\section{Antecedentes e Epidemiologia}

Como afirmado anteriormente, a CPI ainda é uma das doenças mais abundantes em todo o mundo. A incidência de CPI em crianças com dentes decíduos é de 1,76 bilhões (IC 95\%: 1,26 bilhões; 2,39 bilhões) [ 8 ]. Curiosamente, a CPI não se limita a crianças com baixo nível socioeconômico (NSE) [ 9,10 ]. Dados recentes, por exemplo, da Austrália mostram uma prevalência de mais de $50 \%$ de crianças de 6 anos com cárie nos dentes decíduos [ 5 ]. Dados de diferentes partes do mundo mostram até $89,2 \%$ das crianças com ECC no Catar e 36\% na Grécia [ 11,12 ]. Aproximadamente a mesma prevalência (cerca de 40\%) foi relatada nos EUA entre crianças de 2 a 11 anos [13] Um estudo publicado recentemente na Alemanha mostra até $10 \%$ (até $26 \%$ com lesões iniciais) de crianças de 3 anos com CPI e um aumento de até cerca de 50\% em crianças de 6/7 anos [ 14 ]. Embora o índice de ceo-d (dentes obturados perdidos e cariados) tenha diminuído com o tempo em geral [ 10,14 ], a prevalência não diminuiu [ 14 ]. No entanto, um estudo da Alemanha também foi capaz de mostrar diferentes trajetórias e um aumento dos valores de ceo-d quando se olha para uma escala menor em um nível regional [ 10 ]. Enquanto a maioria dos distritos em uma cidade alemã de médio porte mostraram uma diminuição da ceo-d, a ceo- $d$ aumentou em outros distritos ao longo do tempo [10] Milsom et al. descreveram que crianças com uma lesão de cárie já existente têm uma incidência 56 vezes maior de desenvolver novas lesões de cárie em comparação com crianças anteriormente sem cárie [ 15 ]. Problemas de sono e sono insuficiente também podem ser identificados como fator de risco para CPI, pois os problemas de sono levam ao uso mais frequente de mamadeiras com bebidas açucaradas [ 16 - 18 ]. Como o papel dos pais 
ainda não está claro em relação ao desenvolvimento de CPI de seus filhos, vários estudos focaram em diferentes associações [ 19 ]. As origens socioculturais e socioeconômicas dos pais podem ser encontradas como fatores de risco para CLPE, mas o estresse parental não mostra um aumento significativo na CPI com os filhos [ 10, 19 , 20 ]. Não apenas as crianças, mas também seus pais devem ser motivados a cuidar da dentição decídua para prevenir a CPI e, conseqüentemente, o desenvolvimento de cáries na dentição secundária [21].

\section{Etiologia da CPI}

A cárie dentária se desenvolve quando a placa dentária, um biofilme polimicrobiano, não é removida regularmente e a dieta consiste principalmente em monossacarídeos. Os monossacarídeos podem ser metabolizados por muitas das bactérias orais levando a um aumento na produção de ácidos que são capazes de desmineralizar o esmalte [ 22,23 ]. A placa dentária é construída no topo da película começando diretamente após a remoção mecânica do biofilme [ 24 , 25 ]. Mais de 700 espécies / táxons bacterianos são conhecidos na flora oral [26]. Como o habitat oral consiste em muitos nichos ecológicos diferentes, o número relativamente alto de diferentes espécies / táxons pode ser explicado [27] Os microrganismos orais são capazes de interagir uns com os outros e se comunicar principalmente usando o chamado "quorum sensing" (QS) [ 28 ]. Hoje em dia, é bem conhecido que não apenas bactérias, mas também fungos, como Candida albicans e as interações inter-reinos, podem potencializar a progressão da cárie [ 29,30 ]. No entanto, microrganismos cultivados em biofilmes de poliespécies são capazes de produzir exopolissacarídeos (EPS), também conhecidos como substâncias poliméricas extracelulares [ 31 , 32 ]. Com a ajuda do EPS, os microrganismos são capazes de resistir aos antimicrobianos que são usados recentemente em dentifrícios [32] Consequentemente, a formação do biofilme não é interrompida e, juntamente com os sacarídeos absorvidos da dieta, leva a uma placa dentária cariogênica [ 33 ]. A placa dentária no esmalte clinicamente sadio de crianças consiste principalmente de estreptococos e actinomicetos [ 34 ]. Com uma dieta pobre em açúcar, esses microrganismos vivem como comensais em um ambiente homeostático controlando-se uns aos outros [ 35 ]. Assim que açúcares, especialmente alimentos e bebidas açucarados, são consumidos, a microbiota da placa comensal irá absorver esses sacarídeos e metabolizá-los em ácidos, principalmente ácido lático [ 36 ]. Esta produção de ácido leva a uma mudança de $\mathrm{pH}$ de cerca de 7 (neutro) para um $\mathrm{pH}<5,5$ (ácido) [37] Bactérias tolerantes a ácidos, principalmente estreptococos mutans, são capazes de sobreviver a esses ambientes ácidos [ 36 ]. Quando os hábitos de higiene oral e hábitos nutricionais não mudam, uma redução de microrganismos altamente cariogênicos (estreptococos mutans, Candidaspp. E lactobacilos) não pode ser alcançada [ 38 ]. Peterson et al. usou o sequenciamento de última geração (NGS) para identificar a composição microbiana da placa dentária. Eles mostram apenas pequenas diferenças entre os biofilmes coletados de crianças com e sem cárie [39]: Streptococcus 
mitis e Streptococcus sanguinis foram encontrados em ambos os grupos. Estreptococofoi considerado o gênero mais abundante $(>50 \%$ dos microrganismos). Veillonella , Granulicatella , Fusobacterium , Neisseria , Campylobacter , Gemella , Abiotrophia, Selenomonas e Capnocytophaga também foram encontrados em abundância entre 1 e $10 \%$ do biofilme [39]. Simon-Soro et al. também detectou espécies de Lactobacillus, conhecidas como bactérias resistentes ao ácido, associadas à cárie $[40,41]$ Embora os estudos descritos acima usem estratégias de NGS, essa técnica está se desenvolvendo rapidamente e estudos recentes são capazes de usar modelos ainda mais sofisticados de previsão de CPI [ 42 ]. Teng et al. usaram amostras in vivo de um estudo de coorte de 3 anos e mostraram, com a ajuda de modelagem matemática, queS. mutans não era o principal causador de cárie, mas identificou Veillonella spp. e Prevotella spp. em vez disso [ 43 ]. Veillonella atypical , V. dispar e $V$. parvula, bem como Prevotella spp. foram identificados como bactérias que são principalmente responsáveis pelo desenvolvimento de ECC [43 ].

Em conclusão, a CPI se desenvolve assim que a placa dentária não é removida adequadamente e uma dieta açucarada, especialmente alimentos e bebidas adoçados, é consumida. Isso leva a uma alteração do metabolismo com a microbiota da placa dentária, produzindo principalmente ácidos lácticos que irão desmineralizar o esmalte. Prevotella spp. e Veillonella spp. mostraram ser fatores de risco microbianos, enquanto junto com os fungos, as bactérias podem desencadear metabolismos ácidos e virulência dos microrganismos [ 29, 30,41 - 43 ].

\section{Características do esmalte decíduo e esmalte de dentes permanentes}

O esmalte é o tecido mais duro do corpo humano. Consiste principalmente em hidroxiapatita (97\%) (HAP), Ca ${ }_{5}\left(\mathrm{PO}_{4}\right)_{3}(\mathrm{OH})$, que é um mineral de fosfato de cálcio [44-49]. O esmalte é altamente mineralizado e tem propriedades mecânicas extraordinárias [ $44,45,47,50,51$ ] . O interior de um dente consiste em dentina (cerca de $70 \%$ de HAP e $20 \%$ de proteínas, principalmente colágeno e $10 \%$ de água), produzida pelos odontoblastos, e o esmalte, que é formado por ameloblastos. Os ameloblastos estão restritos a produzir esmalte uma vez: os ameloblastos produzem várias proteínas e atraem íons de cálcio e fosfato para cristalizá-los [52, 53 ]. O esmalte dos dentes decíduos é construído em um período significativamente mais curto (24 meses) do que os dentes permanentes (até 16 anos) [52]. A conseqüência do menor tempo para o desenvolvimento do esmalte é a formação de um esmalte muito fino (metade da espessura da dos dentes permanentes) e uma microestrutura menos organizada [ 54, 55 ]. Como consequência, os ácidos são capazes de desmineralizar o esmalte decíduo mais rápido do que o esmalte permanente [ 56 - 58 ].

\section{Fatores de risco}

A CPI é conhecida por ser uma doença multifatorial. Alimentos e bebidas açucarados podem levar a um estado disbiótico da composição microbiana, causando 
cáries. Como a CPI também é conhecida como "cárie de mamadeira", as práticas de alimentação são percebidas como o principal fator de risco para o desenvolvimento de CPI [ $9,59,60$ ]. Aqui, os incisivos e molares superiores são afetados primeiro, seguidos pelos molares da mandíbula inferior e, finalmente, os incisivos da mandíbula inferior [ 61 ]. Crianças que dormem com mamadeiras com chá adoçado ou leite contendo vários açúcares cariogênicos apresentam alto risco de desenvolver CPI. Como consequência de beber durante a noite, sem depuração de açúcares, as bactérias orais irão produzir ácido láctico rapidamente, desmineralizando o esmalte [ 9,62 ]. Hoje em dia, não apenas as mamadeiras, mas também vários outros sucos adoçados consumidos ao longo do dia ou mesmo à noite aumentam o risco de desenvolver cáries. A CPI é uma doença que afeta famílias com baixo SES e famílias com alto SES [ 9,10 ]. No entanto, o desemprego e o histórico de migração podem ser considerados fatores de risco para disparidades espaciais na CPI [ 10 ]. Outros fatores importantes que aumentam o risco de desenvolver CPI são a escovação irregular (remoção mecânica da placa) e / ou a escovação sem supervisão de qualquer cuidador [ 63 ]. Portanto, a escovação completa supervisionada dos dentes duas vezes ao dia deve ser aplicada [ 64 ].

\section{Recomendações Gerais}

A dentição decídua geralmente irrompe 6 a 8 meses após o nascimento [ 65 ]. Como a cavidade oral é altamente sensível, toques suaves na mucosa oral e gengiva devem ser realizados no início da vida infantil para acostumá-los à escovação dos dentes. A escovação de pelo menos dois a três minutos deve ser realizada duas vezes ao dia pelos cuidadores assim que o primeiro dente irromper [ 65 ]. A maioria dos dentistas recomenda o uso de uma quantidade do tamanho de uma ervilha de creme dental com flúor para crianças, que geralmente não contém mais de 500 ppm de flúor [ 66 ]. Além disso, géis de flúor podem ser usados [67 ]. No entanto, os efeitos adversos como a fluorose precisam incluir a avaliação dos potenciais efeitos adversos [ 67 ].

\section{Conceitos biomiméticos e escovação dentária para prevenir CPI}

É bem conhecido que os fluoretos e especialmente os cremes dentais fluoretados podem ter um efeito benéfico na inibição da progressão da cárie [ 68 ]. No entanto, uma redução média de cárie de $23 \%$ em comparação com um placebo só pode ser detectada usando dentifrícios contendo um mínimo de 1000 ppm (0,1\%) de flúor. Na Europa, assim como em outras partes do mundo, os cremes dentais para crianças devem conter no máximo 500 ppm (0,05\%) de flúor [ 66,69 ]. Cremes dentais com mais de 1000 ppm de flúor a 1500 ppm de flúor devem ser rotulados na UE como "Crianças de 6 anos ou menos: Use uma quantidade do tamanho de uma ervilha para escovação supervisionada para minimizar a deglutição. No caso de ingestão de flúor de outras fontes, consulte um dentista ou médico "[ 70] Outros países têm restrições e avisos semelhantes. A razão é um risco aumentado de fluorose dentária e fluorose esquelética devido ao acúmulo de flúor de diferentes fontes e ingestão da pasta de dente fluoretada [ 66 ]. Além disso, é discutido se os fluoretos interagem com os ameloblastos e têm impacto negativo na 
formação do esmalte $[53,71]$. Os fluoretos funcionam principalmente devido à aplicação tópica, aumentando a remineralização com íons cálcio e fosfato derivados da saliva [ 72 ]. Consequentemente, a ingestão de comprimidos de flúor e sais fluoretados são discutidos se devem ser eficazes na proteção contra a cárie ou não [ 66] No passado, era assumido que os fluoretos levavam à formação de fluoroapatita $\left[\mathrm{Ca}_{5}\left(\mathrm{PO}_{4}\right)_{3} \mathrm{~F}\right.$. Esse mecanismo foi pensado para tornar os dentes mais resistentes aos ácidos e proteger o esmalte. No entanto, apenas pequenas quantidades de fluorapatita podem ser detectadas [ 50 , 73 - 75 ]. Curiosamente, os dentes fluoróticos com maior concentração de flúor são ainda menos resistentes aos ácidos do que o esmalte sadio [ 75 - 77 ].

Alternativas para prevenir a cárie e principalmente a CLPE em crianças precisam ser baseadas em estratégias biomiméticas. Vários produtos baseados em diferentes fosfatos de cálcio já estão no mercado e bem estudados [78]. Além de outros, hidroxiapatita (HAP) [Ca $\left.5\left(\mathrm{PO}_{4}\right)_{3}(\mathrm{OH})\right]$ e fosfatos de cálcio amorfo $\left[\mathrm{Ca}_{x}\left(\mathrm{PO}_{4}\right)_{y} \cdot n \mathrm{H}_{2} \mathrm{O}\right.$ ] estabilizados por proteínas de caseína (CPP-ACP) mostram os mais promissores resultados. O HAP foi identificado como muito eficaz na prevenção de ECC em uma coorte de crianças japonesas após um estudo de 3 anos que mostrou uma redução de novas lesões de cárie de até $56 \%[69,79]$ Um estudo clínico duplo-cego randomizado publicado recentemente mostra que a HAP microcristalina não é inferior aos fluoretos na prevenção clínica da cárie [ 80 ]. Além de propriedades remineralizantes que são iguais ao fluoreto de sódio [ 81 ], os microclusters HAP mostraram reduzir a formação de placa dentária in situ e in vivo [ 82 -86]. Lelli et al. foram capazes de observar uma camada protetora na parte superior do esmalte após o uso do creme dental HAP in vivo [87]. Resultados semelhantes podem ser encontrados ao usar o CPPACP. Este fosfato de cálcio também é capaz de remineralizar lesões de esmalte iniciais equivalentes a fluoretos [88] Diferentes estudos mostraram que lesões precoces também podem ser remineralizadas e regredidas usando CPP-ACP [ 89 ] e, em relação à remineralização, identificou o CPP-ACP como sendo superior a um produto contendo alto teor de flúor (5000 ppm de flúor) [ 90 ]. No entanto, outros estudos mostraram resultados contrários [ 91,92 ].

Além de remineralizar os ingredientes, os cremes dentais devem ter propriedades de controle do biofilme que não afetem a saúde das crianças [ 93 ] (Tabela 1 ). 
Tabela 1: Ingredientes principais para cremes dentais infantis (até 6 anos).

Abrasivos à base de carbonato de sílica ou cálcio com baixo valor RDA (RDA: abrasão de dentina radioativa)

Hidroxiapatita, ACP-CPP, fluoretos em baixas concentraçōes

O uso de um creme dental apropriado deve ser acompanhado por escovação supervisionada / apoiada duas vezes ao dia, bem como visitas regulares ao dentista (pelo menos uma vez por ano) [ 96 ]. No que diz respeito às habilidades motoras de crianças muito pequenas, a escovagem deve ser realizada com escovas elétricas ou escovas de dente manuais feitas especialmente para crianças sob supervisão dos pais [ 97 ]. Embora existam várias técnicas de escovação conhecidas, para crianças mais novas, a técnica de escovação horizontal é recomendada combinada com um período de escovação de 3 minutos [98] Os pediatras também devem verificar a saúde bucal e a anamnese de flúor das crianças, ao mesmo tempo em que perguntam aos pais sobre a higiene bucal da criança. As escovas de dentes devem ser substituídas a cada 3 meses ou quando as cerdas se desgastarem com o uso [ 99 ].

\section{CONCLUSÃO}

Além de uma dieta com baixo teor de açúcar, as crianças devem escovar os dentes duas vezes ao dia sob a supervisão dos pais e receber ajuda com a escovação. Os cuidadores devem apoiar especialmente crianças muito pequenas (com menos de 3 anos) continuamente. Os dentifrícios devem conter principalmente agentes remineralizantes promissores para a higiene bucal de crianças, como fosfatos de cálcio.

\section{THE AUTHORS DECLARE NO CONFLICTS OF INTEREST.}

\section{DECLARAÇÃO CCBY}

Este artigo é uma cópia com adaptação para o português do original “Meyer, F., and J. Enax. "Early childhood caries: epidemiology, aetiology, and prevention." International journal of dentistry 2018 (2018)." 100

Esta cópia com adaptação para o português teve como alteração além do idioma a autoria do artigo.

Os autores da publicação original não deram endosso específico a esta cópia com 
autorização para a português, a não ser a licença CCBY 4.0 disponibilizada pelo periódico que publicou o artigo original.

Este é o link da licença: https://creativecommons.org/licenses/by/4.0/

Este é o link do artigo original: https://www.hindawi.com/journals/ijd/2018/1415873/

\section{REFERÊNCIAS}

1. R. Naidu, J. Nunn, and E. Donnelly-Swift, "Oral health-related quality of life and early childhood caries among preschool children in Trinidad," BMC Oral Health, vol. 16, no. 1, p. 128, 2016.View at: Publisher Site I Google Scholar

2. S. L. Filstrup, D. Briskie, M. da Fonseca, L. Lawrence, A. Wandera, and M. R. Inglehart, "Early childhood caries and quality of life: child and parent perspectives," Pediatric Dentistry, vol. 25, no. 5, pp. 431-440, 2003.View at: Google Scholar

3. J. Abanto, T. S. Carvalho, F. M. Mendes, M. T. Wanderley, M. Bonecker, and D. P. Raggio, "Impact of oral diseases and disorders on oral health-related quality of life of preschool children," Community Dentistry and Oral Epidemiology, vol. 39, no. 2, pp. 105-114, 2011.View at: Publisher Site I Google Scholar

4. P. S. Casamassimo, S. Thikkurissy, B. L. Edelstein, and E. Maiorini, "Beyond the dmft: the human and economic cost of early childhood caries," Journal of the American Dental Association, vol. 140, no. 6, pp. 650-657, 2009.View at: $\underline{\text { Publisher Site I Google Scholar }}$

5. S. Chrisopoulos and J. E. Harford, Oral Health and Dental Care in Australia: Key Facts and Figures 2015, Australian Institute of Health and Welfare and the University of Adelaide, Canberra, ACT, Australia, 2016.

6. A. J. Righolt, M. Jevdjevic, W. Marcenes, and S. Listl, "Global-, regional-, and country-level economic impacts of dental diseases in 2015," Journal of Dental Research, vol. 97, no. 5, pp. 501-507, 2018.View at: Publisher Site I Google Scholar

7. A. BaniHani, C. Deery, J. Toumba, T. Munyombwe, and M. Duggal, "The impact of dental caries and its treatment by conventional or biological approaches on the oral healthrelated quality of life of children and carers," International Journal of Paediatric Dentistry, vol. 28, no. 2, pp. 266-276, 2017.View at: Publisher Site I Google Scholar

8. T. Vos, A. A. Abajobir, K. H. Abate et al., "Global, regional, and national incidence, prevalence, and years lived with disability for 328 diseases and injuries for 195 countries, 1990-2016: a systematic analysis for the Global Burden of Disease Study," The Lancet, vol. 390, no. 10100, pp. 1211-1259, 2016.View at: Publisher Site I Google Scholar

9. H. Colak, C. T. Dulgergil, M. Dalli, and M. M. Hamidi, "Early childhood caries update: a review of causes, diagnoses, and treatments," Journal of Natural Science, Biology, and 
Medicine, vol. 4, no. 1, pp. 29-38, 2013.View at: $\underline{\text { Publisher Site I Google Scholar }}$

10. F. Meyer, A. Karch, K. M. Schlinkmann et al., "Sociodemographic determinants of spatial disparities in early childhood caries: an ecological analysis in Braunschweig, Germany," Community Dentistry and Oral Epidemiology, vol. 45, no. 5, pp. 442-448, 2017.View at: Publisher Site | Google Scholar

11. A. Alkhtib, A. Ghanim, M. Temple-Smith, L. B. Messer, M. Pirotta, and M. Morgan, "Prevalence of early childhood caries and enamel defects in four and five-year old Qatari preschool children," BMC Oral Health, vol. 16, p. 73, 2016.View at: $\underline{\text { Publisher Site I Google }}$ $\underline{\text { Scholar }}$

12. C. J. Oulis, K. Tsinidou, G. Vadiakas, E. Mamai-Homata, A. Polychronopoulou, and T. Athanasouli, "Caries prevalence of 5, 12 and 15-year-old Greek children: a national pathfinder survey," Community Dental Health, vol. 29, no. 1, pp. 29-32, 2012.View at: Google Scholar

13. B. A. Bugis, "Early childhood caries and the impact of current U.S. Medicaid program: an overview," International Journal of Dentistry, vol. 2012, Article ID 348237, 7 pages, 2012.View at: Publisher Site | Google Scholar

14. R. Basner, R. M. Santamaría, J. Schmoeckel, E. Schüler, and C. Splieth, Epidemiologische Begleituntersuchungen zur Gruppenprophylaxe 2016, DAJ-Deutsche Arbeitsgemeinschaft für Jugendzahnpflege e. V, Bonn, Germany, 2018.

15. K. M. Milsom, A. S. Blinkhorn, and M. Tickle, "The incidence of dental caries in the primary molar teeth of young children receiving National Health Service funded dental care in practices in the North West of England," British Dental Journal, vol. 205, p. E14, 2008.View at: $\underline{\text { Publisher Site I Google Scholar }}$

16. S. D. Shantinath, D. Breiger, B. J. Williams, and J. E. Hasazi, "The relationship of sleep problems and sleep-associated feeding to nursing caries," Pediatric Dentistry, vol. 18, no. 5, pp. 375-378, 1996.View at: Google Scholar

17. H. Chen, S. Tanaka, K. Arai, S. Yoshida, and K. Kawakami, "Insufficient sleep and incidence of dental caries in deciduous teeth among children in Japan: a population-based cohort study," Journal of Pediatrics, pii: S0022-3476(18)30380-9, 2018.View at: $\underline{\text { Publisher }}$ Site | Google Scholar

18. I. Kraljevic, C. Filippi, and A. Filippi, "Risk indicators of early childhood caries (ECC) in children with high treatment needs," Swiss Dental Journal, vol. 127, no. 5, pp. 398-410, 2017.View at: Google Scholar

19. M. Hooley, H. Skouteris, C. Boganin, J. Satur, and N. Kilpatrick, "Parental influence and the development of dental caries in children aged 0-6 years: a systematic review of the 
literature," Journal of Dentistry, vol. 40, no. 11, pp. 873-885, 2012.View at: $\underline{\text { Publisher }}$ Site I Google Scholar

20. S. E. Jabbarifar, N. Ahmady, S. A. R. Sahafian, F. Samei, and S. Soheillipour, "Association of parental stress and early childhood caries," Dental Research Journal, vol. 6, no. 2, pp. 6570, 2009.View at: Google Scholar

21. K. Narksawat, A. Boonthum, and U. Tonmukayakul, "Roles of parents in preventing dental caries in the primary dentition among preschool children in Thailand," Asia-Pacific Journal of Public Health, vol. 23, no. 2, pp. 209-216, 2011.View at: $\underline{\text { Publisher Site I Google Scholar }}$

22. V. Zijnge, M. B. van Leeuwen, J. E. Degener et al., "Oral biofilm architecture on natural teeth," PLoS One, vol. 5, no. 2, Article ID e9321, 2010.View at: Publisher Site I Google $\underline{\text { Scholar }}$

23. F. Meyer and J. Enax, "Die mundhöhle als ökosystem," Biologie in Unserer Zeit, vol. 48, no. 1, pp. 62-68, 2018.View at: Publisher Site I Google Scholar

24. B. Rosan and R. J. Lamont, "Dental plaque formation," Microbes and Infection, vol. 2, no. 13, pp. 1599-1607, 2000.View at: $\underline{\text { Publisher Site I Google Scholar }}$

25. R. Huang, M. Li, and R. L. Gregory, "Bacterial interactions in dental biofilm," Virulence, vol. 2, no. 5, pp. 435-444, 2011.View at: Publisher Site I Google Scholar

26. D. Verma, P. K. Garg, and A. K. Dubey, "Insights into the human oral microbiome," Archives of Microbiology, vol. 200, no. 4, pp. 525-540, 2018.View at: $\underline{\text { Publisher Site I Google }}$ Scholar

27. X. Xu, J. He, J. Xue et al., "Oral cavity contains distinct niches with dynamic microbial communities," Environmental Microbiology, vol. 17, no. 3, pp. 699-710, 2015.View at: $\underline{\text { Publisher Site I Google Scholar }}$

28. Y.-H. Li and X. Tian, "Quorum sensing and bacterial social interactions in biofilms," Sensors, vol. 12, no. 3, pp. 2519-2538, 2012.View at: Publisher Site I Google Scholar

29. H. Sztajer, S. P. Szafranski, J. Tomasch et al., "Cross-feeding and interkingdom communication in dual-species biofilms of Streptococcus mutans and Candida albicans," ISME Journal, vol. 8, no. 11, pp. 2256-2271, 2014.View at: $\underline{\text { Publisher }}$ Site I Google Scholar

30. M. L. Falsetta, M. I. Klein, P. M. Colonne et al., "Symbiotic relationship between Streptococcus mutans and Candida albicans synergizes virulence of plaque biofilms in vivo," Infection and Immunity, vol. 82, no. 5, pp. 1968-1981, 2014.View at: $\underline{\text { Publisher Site I Google Scholar }}$ 
31. T. T. More, J. S. Yadav, S. Yan, R. D. Tyagi, and R. Y. Surampalli, "Extracellular polymeric substances of bacteria and their potential environmental applications," Journal of Environmental Management, vol. 144, pp. 1-25, 2014.View at: Publisher Site I Google Scholar

32. H. Koo, M. L. Falsetta, and M. I. Klein, "The exopolysaccharide matrix: a virulence determinant of cariogenic biofilm," Journal of Dental Research, vol. 92, no. 12, pp. 10651073, 2013.View at: Publisher Site | Google Scholar

33. P. Lingstrom, F. O. van Ruyven, J. van Houte, and R. Kent, "The pH of dental plaque in its relation to early enamel caries and dental plaque flora in humans," Journal of Dental Research, vol. 79, no. 2, pp. 770-777, 2000.View at: Publisher Site I Google Scholar

34. N. Takahashi and B. Nyvad, "The role of bacteria in the caries process: ecological perspectives," Journal of Dental Research, vol. 90, no. 3, pp. 294-303, 2011.View at: Publisher Site | Google Scholar

35. M. Kilian, I. L. C. Chapple, M. Hannig et al., "The oral microbiome-an update for oral healthcare professionals," British Dental Journal, vol. 221, no. 10, pp. 657-666, 2016.View at: Publisher Site I Google Scholar

36. R. Touger-Decker and C. van Loveren, "Sugars and dental caries," American Journal of Clinical Nutrition, vol. 78, no. 4, pp. 881S-892S, 2003.View at: Publisher Site I Google Scholar

37. I. Struzycka, "The oral microbiome in dental caries," Polish Journal of Microbiology, vol. 63, no. 2, pp. 127-135, 2014.View at: Google Scholar

38. T. Klinke, M. Urban, C. Luck, C. Hannig, M. Kuhn, and N. Kramer, "Changes in Candida spp., mutans streptococci and lactobacilli following treatment of early childhood caries: a 1year follow-up," Caries Research, vol. 48, no. 1, pp. 24-31, 2014.View at: Publisher Site | Google Scholar

39. S. N. Peterson, T. Meissner, A. I. Su et al., "Functional expression of dental plaque microbiota," Frontiers in Cellular and Infection Microbiology, vol. 4, p. 108, 2014.View at: $\underline{\text { Publisher Site I Google Scholar }}$

40. A. Simon-Soro, I. Tomas, R. Cabrera-Rubio, M. D. Catalan, B. Nyvad, and A. Mira, "Microbial geography of the oral cavity," Journal of Dental Research, vol. 92, no. 7, pp. 616-621, 2013.View at: Publisher Site I Google Scholar

41. A. Simon-Soro and A. Mira, "Solving the etiology of dental caries," Trends in Microbiology, vol. 23, no. 2, pp. 76-82, 2015.View at: Publisher Site I Google Scholar

42. E. Hajishengallis, Y. Parsaei, M. I. Klein, and H. Koo, "Advances in the microbial etiology and pathogenesis of early childhood caries," Cell Host \& Microbe, vol. 32, no. 1, pp. 24- 
34, 2017.View at: Publisher Site | Google Scholar

43. F. Teng, F. Yang, S. Huang et al., "Prediction of early childhood caries via spatial-temporal variations of oral microbiota," Cell Host \& Microbe, vol. 18, no. 3, pp. 296-306, 2015.View at: $\underline{\text { Publisher Site I Google Scholar }}$

44. S. V. Dorozhkin and M. Epple, "Biological and medical significance of calcium phosphates," Angewandte Chemie International Edition, vol. 41, no. 17, pp. 3130-3146, 2002.View at: Publisher Site | Google Scholar

45. J. Enax and M. Epple, "Synthetic hydroxyapatite as a biomimetic oral care agent," Oral Health \& Preventive Dentistry, vol. 16, no. 1, pp. 7-19, 2018.View at: $\underline{\text { Publisher }}$ Site I Google Scholar

46. P. W. Brown and B. Constantz, Hydroxyapatite and Related Materials, CRC Press, Boca Raton, FL, USA, 1994.

47. H. A. Lowenstam and S. Weiner, On Biomineralization, Oxford University Press, Oxford, UK, 1989.

48. M. F. Teaford, M. M. Smith, and M. W. J. Ferguson, Development, Function and Evolution of Teeth, Cambridge University Press, Cambridge, UK, 2000.

49. M. S. Tung and D. Skrtic, "Interfacial properties of hydroxyapatite, fluoroapatite and octacalcium phosphate," Monographs in Oral Science, vol. 18, pp. 112-129, 2001.View at: $\underline{\text { Publisher Site I Google Scholar }}$

50. J. Enax, O. Prymak, D. Raabe, and M. Epple, "Structure, composition, and mechanical properties of shark teeth," Journal of Structural Biology, vol. 178, no. 3, pp. 290-299, 2012.View at: Publisher Site I Google Scholar

51. E. D. Yilmaz, S. Bechtle, H. Özcoban, A. Schreyer, and G. A. Schneider, "Fracture behavior of hydroxyapatite nanofibers in dental enamel under micropillar compression," Scripta Materialia, vol. 68, no. 6, pp. 404-407, 2013.View at: Publisher Site I Google Scholar

52. K.-J. Moll and M. Moll, Kurzlehrbuch Anatomie, Elsevier, New York, NY, USA, 2000.

53. C. E. Smith, R. Wazen, Y. Hu et al., "Consequences for enamel development and mineralization resulting from loss of function of ameloblastin or enamelin," European Journal of Oral Sciences, vol. 117, no. 5, pp. 485-497, 2009.View at: $\underline{\text { Publisher }}$ Site I Google Scholar

54. M. A. De Menezes Oliveira, C. P. Torres, J. M. Gomes-Silva et al., "Microstructure and mineral composition of dental enamel of permanent and deciduous teeth," Microscopy Research and Technique, vol. 73, no. 5, pp. 572-577, 2010.View at: Google Scholar

55. A. Lucchese and E. Storti, "Morphological characteristics of primary enamel surfaces 
versus permanent enamel surfaces: SEM digital analysis," European Journal of Paediatric Dentistry, vol. 12, no. 3, pp. 179-183, 2011.View at: Google Scholar

56. P. R. Wilson and A. D. Beynon, "Mineralization differences between human deciduous and permanent enamel measured by quantitative microradiography," Archives of Oral Biology, vol. 34, no. 2, pp. 85-88, 1989.View at: Publisher Site I Google Scholar

57. M. C. Z. Alcantara-Galeana, R. Contreras-Bulnes, L. E. Rodríguez-Vilchis et al., "Microhardness, structure, and morphology of primary enamel after phosphoric acid, selfetching adhesive, and Er:YAG laser etching," International Journal of Optics, vol. 2017, Article ID 7634739, 8 pages, 2017.View at: $\underline{\text { Publisher Site | Google Scholar }}$

58. C. M. Zamudio-Ortega, R. Contreras-Bulnes, R. J. Scougall-Vilchis, R. A. Morales-Luckie, O. F. Olea-Mejia, and L. E. Rodriguez-Vilchis, "Morphological, chemical and structural characterisation of deciduous enamel: SEM, EDS, XRD, FTIR and XPS analysis," European Journal of Paediatric Dentistry, vol. 15, no. 3, pp. 275-280, 2014.View at: Google Scholar

59. S. Anil and P. S. Anand, "Early childhood caries: prevalence, risk factors, and prevention," Frontiers in Pediatrics, vol. 5, p. 157, 2017.View at: $\underline{\text { Publisher Site I Google }}$ Scholar

60. C. A. Feldens, P. H. Rodrigues, G. de Anastacio, M. R. Vitolo, and B. W. Chaffee, "Feeding frequency in infancy and dental caries in childhood: a prospective cohort study," International Dental Journal, vol. 68, no. 2, pp. 113-121, 2017.View at: Publisher Site I Google Scholar

61. A. H. Wyne, "Early childhood caries: nomenclature and case definition," Community Dentistry and Oral Epidemiology, vol. 27, no. 5, pp. 313-315, 1999.View at: $\underline{\text { Publisher }}$ Site I Google Scholar

62. W. M. Avila, I. A. Pordeus, S. M. Paiva, and C. C. Martins, "Breast and bottle feeding as risk factors for dental caries: a systematic review and meta-analysis," PLoS One, vol. 10, no. 11, Article ID e0142922, 2015.View at: $\underline{\text { Publisher Site I Google Scholar }}$

63. P. Prakash, P. Subramaniam, B. H. Durgesh, and S. Konde, "Prevalence of early childhood caries and associated risk factors in preschool children of urban Bangalore, India: a crosssectional study," European Journal of Dentistry, vol. 6, no. 2, pp. 141-152, 2012.View at: Google Scholar

64. R. J. Berkowitz, "Causes, treatment and prevention of early childhood caries: a microbiologic perspective," Journal of the Canadian Dental Association, vol. 69, no. 5, pp. 304-307, 2003.View at: Google Scholar

65. "Tooth eruption," Journal of the American Dental Association, vol. 137, no. 1, p. 127, 2006.View at: Publisher Site | Google Scholar 
66. H. Limeback and C. Robinson, "Fluoride therapy," in Comprehensive Preventive Dentistry, pp. 251-282, John Wiley \& Sons, Ltd., Hoboken, NY, USA, 2012.View at: Google Scholar

67. V. C. Marinho, H. V. Worthington, T. Walsh, and L. Y. Chong, "Fluoride gels for preventing dental caries in children and adolescents," Cochrane Database of Systematic Reviews, vol. 6, p. CD002280, 2015.View at: Publisher Site I Google Scholar

68. T. Walsh, H. V. Worthington, A. M. Glenny, P. Appelbe, V. C. Marinho, and X. Shi, "Fluoride toothpastes of different concentrations for preventing dental caries in children and adolescents," Cochrane Database of Systematic Reviews, vol. 1, p. Cd007868, 2010.View at: $\underline{\text { Publisher Site I Google Scholar }}$

69. C. V. Loveren, Toothpastes, vol. 23, Karger Publishers, Basel, Switzerland, 2013.

70. European Academy of Paediatric Dentistry, "Guidelines on the use of fluoride in children: an EAPD policy document," European Archives of Paediatric Dentistry, vol. 10, no. 3, pp. 129-135, 2009.View at: Google Scholar

71. A. L. Bronckers, D. M. Lyaruu, and P. K. DenBesten, "The impact of fluoride on ameloblasts and the mechanisms of enamel fluorosis," Journal of Dental Research, vol. 88, no. 10, pp. 877-893, 2009.View at: Publisher Site | Google Scholar

72. J. M. ten Cate, "Review on fluoride, with special emphasis on calcium fluoride mechanisms in caries prevention," European Journal of Oral Sciences, vol. 105, no. 5, pp. 461-465, 1997.View at: Publisher Site | Google Scholar

73. J. A. Weatherell, C. Robinson, and A. S. Hallsworth, "Changes in the fluoride concentration of the labial enamel surface with age," Caries Research, vol. 6, no. 4, pp. 312-324, 1972.View at: Publisher Site I Google Scholar

74. F. Muller, C. Zeitz, H. Mantz et al., "Elemental depth profiling of fluoridated hydroxyapatite: saving your dentition by the skin of your teeth?" Langmuir, vol. 26, no. 24, pp. 18750-18759, 2010.View at: Google Scholar

75. F. Neues, A. Klocke, F. Beckmann, J. Herzen, J. P. Loyola-Rodriguez, and M. Epple, "Mineral distribution in highly fluorotic and in normal teeth: a synchrotron microcomputer tomographic study," Materialwissenschaft und Werkstofftechnik, vol. 40, no. 4, pp. 294296, 2009.View at: Publisher Site I Google Scholar

76. L. M. Marin, J. A. Cury, L. M. Tenuta, J. E. Castellanos, and S. Martignon, "Higher fluorosis severity makes enamel less resistant to demineralization," Caries Research, vol. 50, no. 4, pp. 407-413, 2016.View at: Publisher Site I Google Scholar

77. B. Ogaard, "Effects of fluoride on caries development and progression in vivo," Journal of Dental Research, vol. 69, pp. 813-819, 1990.View at: Publisher Site I Google Scholar 
78. S. V. Dorozhkin, "Calcium orthophosphates (CaPo4) and dentistry," Bioceramics Development and Applications, vol. 6, no. 96, 2016.View at: Google Scholar

79. K. Kani, M. Kani, A. Isozaki, H. Shintani, T. Ohashi, and T. Tokumoto, "Effect of apatitecontaining dentifrices on dental caries in school children," Journal of Dental Health, vol. 39, no. 1, pp. 104-109, 1989.View at: Publisher Site I Google Scholar

80. U. Schlagenhauf, K.-H. Kunzelmann, C. Hannig et al., "Microcrystalline hydroxyapatite is not inferior to fluorides in clinical caries prevention: a randomized, double-blind, noninferiority trial," bioRxiv, 2018.View at: $\underline{\text { Publisher Site I Google Scholar }}$

81. K. Najibfard, K. Ramalingam, I. Chedjieu, and B. T. Amaechi, "Remineralization of early caries by a nano-hydroxyapatite dentifrice," Journal of Clinical Dentistry, vol. 22, no. 5, pp. 139-143, 2011.View at: Google Scholar

82. A. Kensche, C. Holder, S. Basche, N. Tahan, C. Hannig, and M. Hannig, "Efficacy of a mouthrinse based on hydroxyapatite to reduce initial bacterial colonisation in situ," Archives of Oral Biology, vol. 80, pp. 18-26, 2017.View at: $\underline{\text { Publisher Site I Google }}$ Scholar

83. I. Harks, Y. Jockel-Schneider, U. Schlagenhauf et al., "Impact of the daily use of a microcrystal hydroxyapatite dentifrice on de novo plaque formation and clinical/microbiological parameters of periodontal health. A randomized trial," PLoS One, vol. 11, Article ID e0160142, 2016.View at: Publisher Site I Google Scholar

84. C. Hannig, S. Basche, T. Burghardt, A. Al-Ahmad, and M. Hannig, "Influence of a mouthwash containing hydroxyapatite microclusters on bacterial adherence in situ," Clinical Oral Investigations, vol. 17, no. 3, pp. 805-814, 2013.View at: $\underline{\text { Publisher }}$ Site I Google Scholar

85. C. Palmieri, G. Magi, G. Orsini, A. Putignano, and B. Facinelli, "Antibiofilm activity of zinccarbonate hydroxyapatite nanocrystals against Streptococcus mutans and mitis group Streptococci," Current Microbiology, vol. 67, no. 6, pp. 679-681, 2013.View at: $\underline{\text { Publisher }}$ Site I Google Scholar

86. S. A. Hegazy and I. R. Salama, "Antiplaque and remineralizing effects of Biorepair mouthwash: a comparative clinical trial," Pediatric Dental Journal, vol. 26, no. 3, pp. 8994, 2016.View at: Publisher Site I Google Scholar

87. M. Lelli, M. Marchetti, I. Foltran et al., "Remineralization and repair of enamel surface by biomimetic Zn-carbonate hydroxyapatite containing toothpaste: a comparative in vivo study," Frontiers in Physiology, vol. 5, p. 333, 2014.View at: Publisher Site I Google $\underline{\text { Scholar }}$

88. J. Li, X. Xie, Y. Wang et al., "Long-term remineralizing effect of casein phosphopeptide- 
amorphous calcium phosphate (CPP-ACP) on early caries lesions in vivo: a systematic review," Journal of Dentistry, vol. 42, no. 7, pp. 769-777, 2014.View at: Publisher Site | Google Scholar

89. D. L. Bailey, G. G. Adams, C. E. Tsao et al., "Regression of post-orthodontic lesions by a remineralizing cream," Journal of Dental Research, vol. 88, no. 12, pp. 1148-1153, 2009.View at: Publisher Site | Google Scholar

90. P. Shen, D. J. Manton, N. J. Cochrane et al., "Effect of added calcium phosphate on enamel remineralization by fluoride in a randomized controlled in situ trial," Journal of Dentistry, vol. 39, no. 7, pp. 518-525, 2011.View at: Publisher Site | Google Scholar

91. N. Gupta, C. Mohan Marya, R. Nagpal, S. Singh Oberoi, and C. Dhingra, "A review of casein phosphopeptide-amorphous calcium phosphate (CPP-ACP) and enamel remineralization," Compendium of Continuing Education in Dentistry, vol. 37, no. 1, pp. 36-39, 2016.View at: Google Scholar

92. Y. Wang, J. Li, W. Sun, H. Li, R. D. Cannon, and L. Mei, "Effect of non-fluoride agents on the prevention of dental caries in primary dentition: a systematic review," PLoS One, vol. 12, no. 8, Article ID e0182221, 2017.View at: Publisher Site I Google Scholar

93. J. M. ten Cate, "The need for antibacterial approaches to improve caries control," Advances in Dental Research, vol. 21, no. 1, pp. 8-12, 2009.View at: Publisher Site I Google Scholar

94. J. Enax and M. Epple, "Die charakterisierung von putzkörpern in zahnpasten," Deutsche Zahnärztliche Zeitung, vol. 73, pp. 116-124, 2018.View at: Google Scholar

95. C. H. Splieth, M. Alkilzy, J. Schmitt, C. Berndt, and A. Welk, "Effect of xylitol and sorbitol on plaque acidogenesis," Quintessence International, vol. 40, no. 4, pp. 279-285, 2009.View at: Google Scholar

96. M. B. Kowash, A. Pinfield, J. Smith, and M. E. Curzon, "Effectiveness on oral health of a long-term health education programme for mothers with young children," British Dental Journal, vol. 188, no. 4, pp. 201-205, 2000.View at: Publisher Site I Google Scholar

97. M. Yaacob, H. V. Worthington, S. A. Deacon et al., "Powered versus manual toothbrushing for oral health," Cochrane Database of Systematic Reviews, vol. 6, 2014.View at: Google Scholar

98. M. Muller-Bolla and F. Courson, "Toothbrushing methods to use in children: a systematic review," Oral Health \& Preventive Dentistry, vol. 11, no. 4, pp. 341-347, 2013.View at: Google Scholar

99. P. M. Glaze and A. B. Wade, "Toothbrush age and wear as it relates to plaque control," Journal of Clinical Periodontology, vol. 13, no. 1, pp. 52-56, 1986.View 
at: Publisher Site | Google Scholar

100. Meyer, F., and J. Enax. "Early childhood caries: epidemiology, aetiology, and prevention." International journal of dentistry 2018 (2018) 